\title{
Cerebral Regulation of Facial Expressions of Pain
}

\author{
Miriam Kunz, ${ }^{1,2}$ Jen-I Chen, ${ }^{1}$ Stefan Lautenbacher, ${ }^{2}$ Etienne Vachon-Presseau, ${ }^{1}$ and Pierre Rainville ${ }^{1}$ \\ ${ }^{1}$ Départment de stomatologie and Centre de recherche de l'Institut universitaire de gériatrie de Montréal, Université de Montréal, Montréal, Quebec H3T \\ 1J4, Canada, and 2Physiological Psychology, Otto-Friedrich University Bamberg, 96045 Bamberg, Germany
}

Facial expression of affective states plays a key role in social interactions. Interestingly, however, individuals differ substantially in their level of expressiveness, ranging from high expressive to stoic individuals. Here, we investigate which brain mechanisms underlie the regulation of facial expressiveness to acute pain. Facial responses, pain ratings, and brain activity (BOLD-fMRI) evoked by noxious heat and warm (control) stimuli were recorded in 34 human volunteers with different degrees of facial expressiveness. Within-subject and between-subject variations in blood oxygenation level-dependent (BOLD) responses were examined specifically in relation to facial responses. Pain expression was inversely related to frontostriatal activity, consistent with a role in downregulating facial displays. More detailed analyses of the peak activity in medial prefrontal cortex revealed negative BOLD responses to thermal stimuli, an effect generally associated with the default mode network. Given that this negative BOLD response was weaker in low expressive individuals during pain, it could reflect stronger engagement in, or reduced disengagement from, self-reflective processes in stoic individuals. The occurrence of facial expressions during pain was coupled with stronger primary motor activity in the face area and-interestingly-in areas involved in pain processing. In conclusion, these results indicate that spontaneous pain expression reflects activity within nociceptive pathways while stoicism involves the active suppression of expression, a manifestation of learned display rules governing emotional communication and possibly related to an increased self-reflective or introspective focus.

\section{Introduction}

Facial expression is a fundamental channel for communication in many species and is well recognized as a critical element regulating social interactions and interpersonal relationships (Darwin, 1872; Ekman, 1999; Halberstadt et al., 2001; Williams, 2002). The encoding of affective states in facial expressions is to a large degree innate (Ekman, 1999), but, nevertheless, the degree to which this channel is actually used to overtly display one's emotions can vary extensively. Facial expressiveness ranges from high expressiveness to stoicism, where no facial expressions are shown despite subjective reports indicating strong emotional experiences (Gross and Levenson, 1993; Richards and Gross, 2000). It has been suggested that the lack of expressiveness reflects a learned inhibitory control, regulated by social norms about when and how one should express emotions (Ekman and Friesen, 1969). Thus, the behavioral expression of emotions would be the "default response" that individuals learn to suppress following social demands. According to this possibility, stoic individuals should show increased activation in frontal brain areas involved in motor inhibition compared with more expressive individuals, in response to affective stimuli.

Received Jan. 13, 2011; revised March 21, 2011; accepted April 20, 2011.

Author contributions: M.K., S.L., and P.R. designed research; M.K., J.-I.C., and E.V.-P. performed research; M.K. and J.-I.C. analyzed data; M.K. and P.R. wrote the paper.

This study was supported by a German TRANSCOOP grant from the Alexander von Humboldt Stiftung (S.L. and P.R.) and a research grant from the Deutsche Forschungsgemeinschaft (Ku2294/2, M.K.). Canadian support was provided by Natural Sciences and Engineering Research Council of Canada (P.R.) grants. We thank Nadine LeBlancfor the support in FACS-coding.

Correspondence should be addressed to Miriam Kunz, Physiological Psychology, University of Bamberg, Markusplatz 3, 96045 Bamberg, Germany. E-mail: miriam.kunz@uni-bamberg.de.

DOI:10.1523/JNEUROSCI.0217-11.2011

Copyright $\odot 2011$ the authors $\quad 0270-6474 / 11 / 318730-09 \$ 15.00 / 0$
Given the important role facial expression plays in social interactions, it is not surprising that many studies have been conducted examining the neural base of communicating affective states via facial expressions. However, while there is a multitude of studies on the brain mechanisms involved in the perception of facial expressions (for reviews, see Tsao and Livingstone, 2008; Fusar-Poli et al., 2009), we know very little about the neural mechanisms underlying the encoding of affective states via facial expressions. The few brain imaging and lesion studies focusing on this topic suggest that there is a network of both subcortical (basal ganglia) and cortical regions (M1, SMA) contributing to the display of spontaneous as well as voluntary facial expressions (Rinn, 1984; Iwase et al., 2002; Blair, 2003; Wild et al., 2006). Although no study so far has focused on the mechanisms underlying spontaneous variations in facial expressiveness, there is nevertheless some evidence pointing to an involvement of frontal regions. Behavioral studies in patients with frontal cortex lesions have suggested an involvement of the frontal cortex in regulating facial expressions (Weddell et al., 1988, 1990; Weddell, 1994; Blair, 2003). In line with this, a recent functional magnetic resonance imaging (fMRI) study in healthy individuals found that the voluntary suppression of facial expressions while watching negative emotion-eliciting films was accompanied by increased activation in prefrontal areas (Goldin et al., 2008).

The aim of the present study was to investigate-for the first time-the brain mechanisms underlying spontaneously occurring variations in facial expressions by studying brain activation (fMRI) associated with the facial encoding of pain experiences in individuals with different degrees of facial expressiveness. Choosing facial encoding of pain has the advantage that comparable levels of subjective pain experience across subjects are relatively easy to 


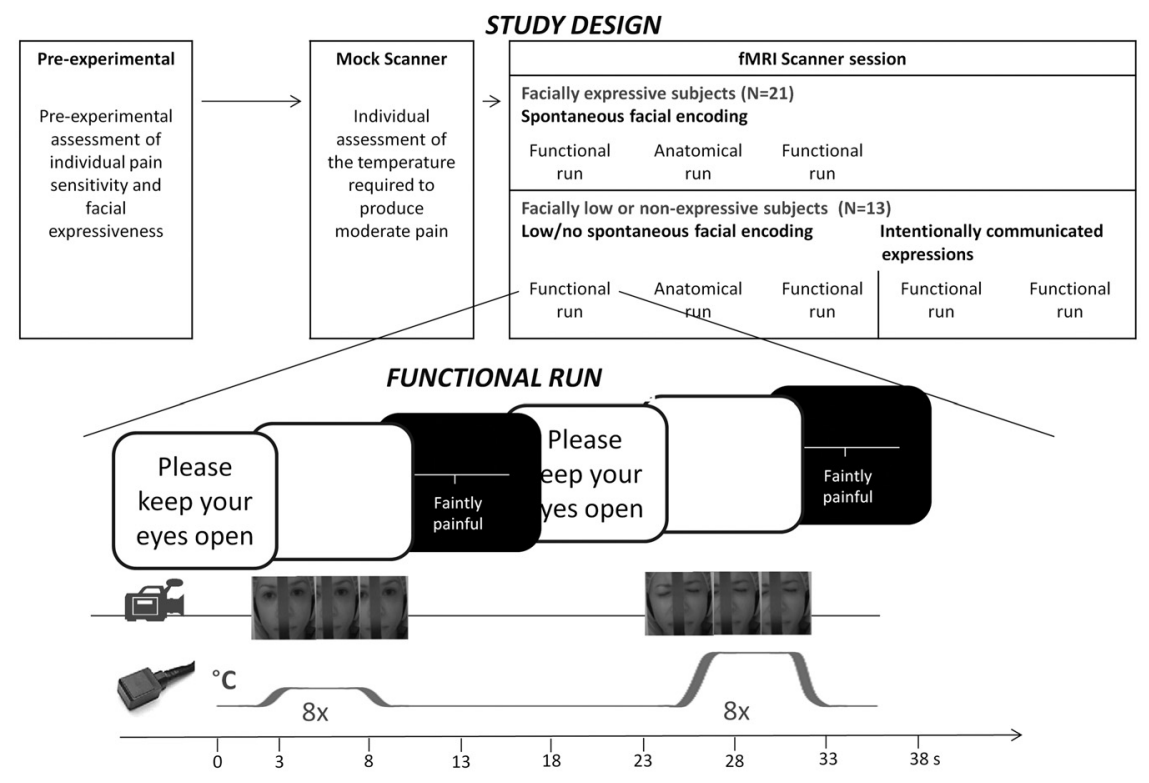

Figure 1. Study design and detailed description of the experimental protocol.

doc). The contact probe was attached to the left lower leg. Baseline temperature was always set to $38^{\circ} \mathrm{C}$. The increase from baseline to destination temperatures was set to take $2 \mathrm{~s}$ with the rising rates adjusted accordingly, depending on the destination temperature (see below). Before subjects entered the scanner, individual pain sensitivity was assessed in a mock MRI scanner (Fig. 1). Since our aim was to investigate the cerebral regulation of facial expressiveness to noxious stimulation, we wanted to exclude variations that are simply due to differences in the subjects' pain experience by evoking comparable levels of pain during the scanning session. To do this, psychophysical magnitude estimations were obtained for each subject to determine the temperature that was perceived as moderately painful (for more details, see Kunz et al., 2008). We selected moderately painful intensities to ensure that painful stimulation would be sufficient to evoke facial responses in most subjects (Kunz et al., 2008), without inducing sensitization or reaching the subject's tolerance. Additionally, a nonpainful control temperature was selected and served as a reference for "baseline" facial expression as well as

elicit, induction can be repeated numerous times without habituation effects, and that even short stimulation times are sufficient to elicit strong experiences.

We hypothesized that low facial expressiveness is accompanied by stronger prefrontal activation consistent with an inhibitory process. We also examined activation of cortical areas known to receive nociceptive inputs from the spinothalamic tract to determine whether differences in facial expression coded at least in part for neural activation within this major ascending pathway.

\section{Materials and Methods}

\section{Participants}

Thirty-four healthy volunteers (female: $N=18$, male: $N=16$ ) between the ages of 18 and 30 years (mean age 23.4 years; SD $=2.5$ ) without history of neurological or psychiatric disease or of chronic pain were recruited for a brain imaging study on pain via advertisements posted on the campuses of the Universite de Montréal and McGill University. Before the brain imaging study, all subjects took part in a $30 \mathrm{~min}$ preexperimental session where the experimental protocol was administered to ensure that thermal pain sensitivity was in a normal range and that individuals with different degrees of facial expressiveness to pain were included. Out of the 34 participants, 13 showed no or only very weak facial responses (facial responses occurred in $\leq 20 \%$ of painful trials) and were classified as facially low/nonexpressive, whereas 21 subjects were classified as facially expressive (facial responses occurred in $>20 \%$ of painful trials). These rates are consistent with our previous studies showing that approximately one third of individuals are facially low or nonexpressive at moderately painful intensities (Kunz et al., 2006, 2008). None of the subjects had taken any analgesic medication or alcohol for at least $24 \mathrm{~h}$ before the test session. All subjects provided written informed consent and received monetary compensation for their participation. The consent form indicated that the aim of the study was to investigate the cerebral response to painful stimulation, and it mentioned that they would be filmed during the experiment, although this was not emphasized at any point during the study [except for the "intentionally expression" condition performed at the end of the experiment in the low/ nonexpressers (Fig. 1)]. The study protocol was approved by the ethics committee of the Centre de recherche de l'Institut universitaire de gériatrie de Montréal.

\section{Material and procedure: thermal stimuli}

Pain was induced experimentally by using a computer-controlled thermal stimulator with a $3 \times 3 \mathrm{~cm}^{2}$ contact probe (Medoc TSA-2001; Me- baseline neural responses to thermal sensory input. Participants were brought into the scanner room for the experiment immediately after.

\section{Material and procedure: assessment of facial, subjective, and brain responses}

In each functional scan, eight nonpainful and eight painful thermal stimuli were applied in a pseudo-random order (Fig. 1). The rate of temperature increase from baseline $\left(38^{\circ} \mathrm{C}\right)$ was adjusted individually to reach the target temperature in $2 \mathrm{~s}$, and remained at a plateau for $5 \mathrm{~s}$, before returning to baseline in $2 \mathrm{~s}$. A long interstimulus interval (18-25 s) was used to prevent sensitization and to allow subjects to rate each stimulus. There were two $8.5 \mathrm{~min}$ functional scans performed, during which we recorded spontaneous facial expressions (Fig. 1). Subjects were instructed to attend to each stimulus, to keep their eyes open, and to try not to move their head and body.

In the 13 facially low/nonexpressive subjects, two additional functional scans were performed. To be able to test whether their lack of spontaneous facial expressions is due to an active expression suppression, we added two runs where low/nonexpressive subjects were explicitly asked to use their face to communicate how they were feeling [runs with intentionally communicated expressions (Fig. 1)]. During those scans, low/nonexpressive subjects were instructed as follows: "Please use your facial expression to communicate as accurately as possible how much pain you feel during the stimulation, without exaggerating your expression, as if your facial expression was the only way you could communicate your experience." These instructions are based on instructions used by Elfenbein et al. (2007).

Facial expression of pain. During all functional scans, the face of the subject was videotaped. This was done using a small MRI-compatible camera (MRC Systems) that was mounted onto the headcoil and captured the face of the subject reflected through a mirror that was attached above the headcoil (image examples are given in Figs. 1). The camera was positioned in a way that it did not block the visual field of the subject. The onset of each thermal stimulus was marked automatically on the video recording using a signal sent from the stimulator to the sound card to identify the occurrence of facial expressions during the stimuli. We quantified facial responses using the Facial Action Coding System [FACS (Ekman and Friesen, 1987)], a fine-grained anatomically based system that is considered the gold standard when decoding facial expressions, including the facial expression of pain. A certified FACS coder identified the frequency and the intensity (5-point scale) of the different action units (AU). As can be seen in Figure 1, a narrow bar blocked the middle part of the face, but with the exception of AU 9 (nose wrinkler) and AU 17 (chin 
Table 1. Facial action units with a critical frequency of occurrence of more than $5 \%$ in painful segments

\begin{tabular}{|c|c|c|c|}
\hline \multirow[b]{2}{*}{ Action unit } & \multirow[b]{2}{*}{ Description } & \multicolumn{2}{|c|}{ Frequency of occurrence } \\
\hline & & Percent $^{a}$ & $\begin{array}{l}\text { Effect size } \\
\text { (Cohen's d) }\end{array}$ \\
\hline $\mathrm{AU} 4^{b}$ & Brow lower & $36.9^{c}$ & $d=1.60^{c}$ \\
\hline $\mathrm{AU} 6 / 7^{b}$ & Orbit tightening & $77.1^{c}$ & $d=1.91^{c}$ \\
\hline AU9/10 ${ }^{b}$ & Levator contraction & $37.3^{c}$ & $d=1.37^{c}$ \\
\hline AU14 & Dimpler & $5.3^{c}$ & $d=0.81^{c}$ \\
\hline AU25/26/27 & Mouth opening & $16.0^{c}$ & $d=0.82^{c}$ \\
\hline$A \cup 43^{b}$ & Eye closure & $18.1^{c}$ & $d=0.75^{c}$ \\
\hline AU45 & Blink & 160.6 & $d=0.12$ \\
\hline
\end{tabular}

Effect sizes for frequency differences between "non-painful" and "painful" segments are given.

${ }^{a}$ Percent denotes the percentage of occurrence in the entire painful segments. Note that this value may exceed $100 \%$ if the response occurred more than once per trial on average.

${ }^{b} \mathrm{AUS}$ s that were more frequent during pain than during nonpainful segments (effect size $d \geq 0.5$ ).

'Medium and strong effect sizes $(d \geq 0.5)$.

raise) (which were more challenging to detect), all other action units were clearly visible. A software designed for the analysis of observational data (the Observer Video-Pro; Noldus Information Technology) was used to segment the videos and to enter the FACS codes into a time-related database. Time segments of $5 \mathrm{~s}$ beginning when the stimulus reached the target temperature were selected for scoring. Pain-relevant AUs were selected based on previous studies (Kunz et al., 2006, 2007, 2008) using the following steps: (1) AUs had to occur in $>5 \%$ of the painful segments recorded, and (2) AUs had to be more frequent during pain than during nonpainful segments (effect size $d \geq 0.5$; these AUs are cross referenced in Table 1 with $b$ ). Following this, mean AU-frequency and mean AUintensity values of the selected AUs were combined (product terms) to form a composite score of pain-relevant facial responses (Kunz et al., 2008). Due to the fact that these composite scores were not distributed normally, ranked composite scores were used for further analyses.

Since this is the first study examining facial expression of pain inside a MRI scanner, we tested whether this restricted environment might have led to inhibition of otherwise spontaneously occurring facial pain displays. The subjects' facial responses assessed during the preexperimental session (Fig. 1) were compared with the facial responses assessed during the scanning session. No significant difference in facial expressiveness was found between these sessions $(p=0.26)$, and test-retest reliability was good and highly significant $(r=0.72, p<0.001)$.

Self-report ratings. After each stimulus, subjects rated the intensity of the stimulus on a computerized visual analog scale (VAS) displayed using E-Prime (Psychology Software Tools) and converted linearly to values between 0 and 100. The scale was labeled with the verbal anchor "faintly painful" in the center so that all nonpainful sensations were rated $<50$ and all painful ones at 50 or above (subjects were instructed that the lower end meant no felt change in the temperature and the upper end indicated extremely strong pain). The VAS was projected to a screen located at the head-end of the scanner, and it could be viewed by the subjects via a mirror attached above the head coil. The ratings were produced by moving a cursor using the index and middle finger of the right hand and were recorded in E-prime. Subjects were not told that the painful and warm temperatures remained constant across trials and runs and were asked to provide rating reflecting pain-felt sensation as accurately as possible.

fMRI image acquisition and analyses. Imaging data were acquired at the Unité de Neuroimagerie Fonctionnelle of the Centre de recherche de l'Institut de gériatrie de Montréal using a 3T Siemens Magneton TIM Trio MRI system with a 12-channel head coil. Participants were placed in a comfortable position and their head stabilized with foam pads and headphones. Earplugs were also given to reduce the noise from the scanner. Scanning consisted of two functional runs [four runs in low/nonexpressive subjects (Fig. 1)], separated by one high-resolution anatomical scan of $9 \mathrm{~min}$. The high resolution $(1 \times 1 \times 1.2 \mathrm{~mm}$ voxel size $)$ T1weighted structural MRI was acquired using a MP-RAGE sequence. A total of 170 whole-brain volumes were acquired during each functional scan using BOLD contrast (Ogawa et al., 1990). Each functional volume comprised 40 interleaved axial slices of $3.40 \mathrm{~mm}$ thickness parallel to the AC-PC line (in-plane resolution $3.44 \times 3.44 \mathrm{~mm}$ ). Volumes were acquired using a gradient echo, echo-planar (EPI) T2*-weighted sequence $\left(\mathrm{TR}=3000 \mathrm{~ms}, \mathrm{TE}=30 \mathrm{~ms}\right.$; flip angle $=90^{\circ}$; matrix; FOV $=$ $220 \times 220 \mathrm{~mm}^{2}$ ).

Image analysis was performed with SPM5 (Statistical Parametric Mapping, Version 5; Wellcome Department of Imaging Neuroscience, London, UK), executed in Matlab 7.4 (MathWorks). Anatomical images were spatially normalized into the MNI space. Functional images were first preprocessed with slice-time correction, and motion corrected by realigning all images to the first image using six-parameter rigid body transformation and reslicing with fourth degree B-spline interpolation. The motion correction parameters were examined for each individual run and led to the exclusion of one run from one subject and some volumes from two subjects ( 20 and $28 \%$ of volumes, respectively). Instantaneous movement was always less than a third of the voxel size in the remaining data (maximum across all subjects and runs: $x=0.571 \mathrm{~mm}$, $y=0.706 \mathrm{~mm}, z=1.07 \mathrm{~mm}$ ), and the amplitude of motion was not significantly related to the occurrence of facial expression during the painful stimulation (i.e., motion correction during pain with facial expression $>$ no facial expression; $p>0.05$, nonsignificant). The BOLD images were then spatially normalized into the MNI space using trilinear interporation, with the normalization parameters determined during the normalization of the structural images. Subsequent spatial smoothing using isotropic $6 \mathrm{~mm}$ full width half maximum Gaussian kernel was applied to the functional images to increase signal-to-noise ratio. A highpass temporal filter (cutoff $=128 \mathrm{~s}$ ) and correction for auto-correlation between successive scans were applied to the time series (AR1).

Warm and painful heat responses were modeled using a canonical hemodynamic response function. Analysis of these data was performed using the general linear model to obtain parameter estimates of stimulus-related activity at each voxel, for each condition, and each subject. At the first level of the analyses, the regressors in the design matrix included the two stimuli and their respective subevents: $W_{\text {arm }}$ ramp-up; Warm, Warm ${ }_{\text {rating }}$; Pain $n_{\text {ramp-up; }}$;

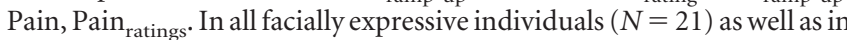
some of the individuals from the low/nonexpressive group who showed slight facial expressions (facial expression occurred in $>10 \%$ and $\leq 20 \%$ of painful trials; $N=7$ ), a second design matrix was built in which pain events were further defined as two separate regressors, namely Pain $_{\text {no expression, }}$ Pain expression $_{\text {, to examine brain activity associated }}$ with facial pain expressions (see below).

Statistical parametric maps of the $t$ statistic $(\operatorname{SPM}\{t\})$ were obtained from linear contrasts between the different regressors (Friston et al., 1995). In the beginning, within-subject contrasts were obtained to look for the overall pain-related brain responses by contrasting the maineffect of Pain $>$ Warm $(\mathrm{P}>\mathrm{W})$.

To investigate the cerebral regulation of facial pain displays, three different analyses were then conducted: (1) We performed a second level between-subject analysis to look for correlations between facial expressiveness (FACS composite ranked scores) and pain-related brain responses obtained from the first level analysis $(\mathrm{P}>\mathrm{W} \times$ FACS ranks). Furthermore, to explore potential interactions between areas related to facial expressiveness, Pearson- $r$ correlations and regressions analyses were computed using b-values obtained in the Pain $>$ Warm contrast and extracted from spherical ROIs ( $6 \mathrm{~mm}$ radius) centered on the peak coordinates defined by the $\mathrm{P}>\mathrm{W} \times$ FACS results.

(2) In the facially expressive group $(N=21)$ as well as in parts of the low/nonexpressive group [slightly expressive individuals $(N=7)$ ], within-subject contrasts were obtained for the differences between painful events with and without spontaneous facial expressions of pain

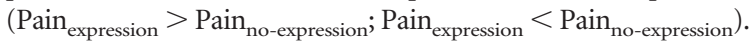

(3) In the low/nonexpressive subjects $(N=13)$, within-subject contrasts were obtained for the differences between functional runs with no or only low spontaneous expressions and runs with intentionally communicated facial expressions of pain (i.e., when instructions were given to express what they felt) ( Pain $_{\text {functional scans 1,2 }}>$ Pain $_{\text {functional runs3,4; }}$; Pain $_{\text {functional scans 1,2 }}<$ Pain $_{\text {functional runs3,4 }}$ ).

A corrected $p$-value of 0.05 (FWE) was used to reveal pain-related brain responses $(\mathrm{P}>\mathrm{W})$. The results of this basic effect of painful stimuli were then established as the a priori areas on which directed searches in 
Table 2. Pain-related BOLD responses across all subjects and conditions (Pain > Warmth)

\begin{tabular}{lllll}
\hline & \multicolumn{2}{l}{ Coordinates } & & $\begin{array}{l}\text { Local peak } \\
t \text { value }\end{array}$ \\
\cline { 2 - 3 } & $x$ & $y$ & $z$ &
\end{tabular}

Targets of the spino-thalamocortical

$$
\text { pathways }
$$$$
\text { S1 }
$$

S2/parietal operculum

ACC

Caudal ACC

$\mathrm{PCC}$

alNS

$\begin{array}{lrr} & 45 & 10 \\ & -38 & 7 \\ \text { pINS/claustrum } & -45 & 14 \\ & 34 & -17 \\ \text { Thalamus } & 41 & -10 \\ & -34 & -17 \\ 14 & -10 \\ & -14 & -14\end{array}$

Motor-related structures

M1

$$
\begin{aligned}
& \text { SMA } \\
& \text { ACC }^{b} \\
& \text { Caudal }^{\text {ACC }}{ }^{b} \\
& \text { Thalamus } \\
& \text { Other areas } \\
& \text { Precuneus } \\
& \text { DLPF }
\end{aligned}
$$

Middle frontal gyrus Inferior frontal gyrus

Parahippocampal gyrus
Substantia nigra

\section{Brainstem}

$\begin{array}{rr}14 & -45 \\ -21 & -45 \\ 58 & -27 \\ 65 & -24 \\ 45 & -24 \\ -55 & -21 \\ 0 & 14 \\ -3 & 0 \\ 0 & -7 \\ 14 & -21 \\ -17 & -31 \\ 34 & 10 \\ 45 & 10 \\ -38 & 7 \\ -45 & 14 \\ 34 & -17 \\ 41 & -10 \\ -34 & -17 \\ 14 & -10 \\ -14 & -14\end{array}$

$\begin{array}{rr}65 & 9.74 \\ 65 & 7.79 \\ 27 & 8.57 \\ 20 & 7.83 \\ 24 & 7.66 \\ 17 & 9.11 \\ 41 & 11.99 \\ 41 & 10.39 \\ 44 & 10.41 \\ 41 & 5.65 \\ 41 & 6.72 \\ 7 & 9.67 \\ 3 & 9.57 \\ 7 & 9.90 \\ -3 & 8.28 \\ 10 & 9.32 \\ -7 & 7.62 \\ 20 & 9.49 \\ 0 & 7.33 \\ 10 & 7.64 \\ & \\ 41 & 5.26^{a} \\ 44 & 6.44 \\ 41 & 5.94 \\ 64 & 10.12\end{array}$

7.66

9.11

11.99

6.72

9.67

9.57

9.90

9.32

9.49

7.33

10.12

$3-7$

\begin{tabular}{rrcl} 
& & & \\
-21 & -72 & 17 & 5.72 \\
31 & 51 & 27 & 5.69 \\
-31 & 45 & 27 & 7.08 \\
-38 & 52 & 20 & 5.59 \\
34 & 28 & 3 & 5.71 \\
45 & 21 & -14 & 5.89 \\
-34 & 28 & 3.4 & 6.42 \\
14 & -10 & -10 & 5.76 \\
10 & -24 & -10 & 7.39 \\
-7 & -28 & -14 & 5.80 \\
7 & -34 & -48 & 6.03 \\
-3 & -34 & -48 & 6.00 \\
\hline
\end{tabular}

Global search threshold: $T=5.58$. $p$-corrected $<0.05$ (FWE); no significant negative peak found at current threshold.

${ }^{a}$ Marginal significance at global threshold.

${ }^{b}$ See the pain-related peaks.

subsequent analyses examining effects of facial expression $(\mathrm{P}>\mathrm{W} \times$ FACS ranks, Pain expression $>$ Pain $_{\text {no-expression }} ;$ Pain $_{\text {runs 1,2 }}<$ Pain $\left._{\text {runs } 3,4}\right)$ were conducted. In these analyses, we first examined pain-related responses within bilateral brain areas commonly activated by painful stimuli (for review, see Apkarian et al., 2005) and targeted by the spinothalamic system in primates [i.e., thalamus, S1, S2, ACC, and INS, SMA (Dum et al., 2009)]. A threshold of $p<0.05$ (one-tail) adjusted using the Bonferroni-correction was applied, based on 12 pain-related regions (bilateral) included in the directed search. The effect of facial expression was also examined in additional global searches using a threshold of $p$-uncorrected $<0.001$.

\section{Results}

\begin{tabular}{|c|c|c|c|c|}
\hline \multirow[b]{2}{*}{ Brain area } & \multicolumn{3}{|c|}{ Coordinates } & \multirow{2}{*}{$\begin{array}{l}\text { Local peak } \\
t \text { value }\end{array}$} \\
\hline & $x$ & $y$ & $z$ & \\
\hline \multicolumn{5}{|c|}{ Targets of the spino-thalamocortical pathways ${ }^{a}$} \\
\hline \multirow[t]{2}{*}{ S1 } & 3 & -34 & 68 & $2.52^{b}$ \\
\hline & -21 & -24 & 68 & $2.44^{b}$ \\
\hline \multirow[t]{2}{*}{ pINS/claustrum } & 34 & -38 & 17 & $3.10^{b}$ \\
\hline & -34 & -10 & 14 & $2.57^{b}$ \\
\hline \multicolumn{5}{|l|}{ Motor-related structures } \\
\hline \multirow[t]{2}{*}{ M1 } & 52 & -7 & 37 & 5.10 \\
\hline & -52 & -10 & 34 & 5.35 \\
\hline SMA & 3 & -17 & 54 & $3.07^{c}$ \\
\hline \multicolumn{5}{|l|}{ Other areas } \\
\hline Posterior cingulate & -14 & -38 & 34 & 3.71 \\
\hline Lingual gyrus & -17 & -72 & 0 & 4.51 \\
\hline \multicolumn{5}{|c|}{ Targets of the "expression suppression" areas ${ }^{d}$} \\
\hline \multirow[t]{2}{*}{ Middle frontal gyrus } & 28 & 45 & 0 & 3.66 \\
\hline & -38 & 45 & -3 & 4.14 \\
\hline \multirow[t]{3}{*}{ MPFC/perigenual ACC } & 0 & 38 & 0 & 4.14 \\
\hline & -14 & 38 & 7 & 3.99 \\
\hline & -14 & 34 & 27 & 3.64 \\
\hline \multicolumn{5}{|l|}{ Motor-related structures } \\
\hline Caudate nucleus & 10 & 7 & 20 & $3.28^{c}$ \\
\hline
\end{tabular}

Pain-related responses

To verify that painful heat stimulation led to different responses than nonpainful heat stimulation (manipulation check), we compared the two stimulation conditions on three indices: self-
Table 3. Between-subject analysis - correlations between facial expressiveness (FACS ranked cores) and brain responses to painful stimulation (Pain $>$ Warm $\times$ FACS rank)

${ }^{a}$ Spontaneous expression: brain responses to painful Heat $>$ Warmth positively correlated with FACS scores. ${ }^{b}$ Directed search threshold at $T=2.84(p=0.05)$. Bonferroni-corrected for 12 pain-related regions. Additional peaks are reported at a global search threshold of $T=3.37, p=0.001$ (uncorrected).

'Marginal significance at global threshold.

${ }^{d}$ Spontaneous nonexpression: brain responses to painful Heat $>$ Warmth negatively correlated with FACS scores.

report ratings, facial expressions, and neural responses. As intended, painful heat stimulation adjusted individually to produce moderate pain (mean temperature: $47.8 \pm 1.0^{\circ} \mathrm{C}$ ) was rated as significantly more intense (mean VAS: $80.1 \pm 7.8$ ) compared with non-painful stimulation (mean temperature: $44.8 \pm 1.1^{\circ} \mathrm{C}$; mean VAS: $30.2 \pm 13.6)(p<0.001)$. As can be seen in Table 1 , painful heat stimulation also elicited increased pain-indicative facial responses compared with nonpainful heat stimulation (including brow lowering, tightening of the muscles surrounding the eyes, and levator contraction, see also examples of facial expressions in Fig. 1). Functional imaging data analysis contrasting the painful and nonpainful stimulation conditions also showed the expected pain-related responses (Table 2). Based on these imaging findings, we selected a priori areas (namely S1, S2, ACC, INS, SMA, and thalamus) for directed search in further analyses (for more details, see Materials and Methods). Overall, our experimental protocol produced reliable perceptual, behavioral, and cerebral responses associated with pain.

\section{Cerebral regulation of facial pain displays}

To investigate the cerebral regulation of facial pain displays, three different analyses were conducted (for more details, see Materials and Methods).

\section{Between-subject analysis (analysis 1)}

Regression analyses were conducted to investigate the relation between facial expressiveness (FACS-ranked composite scores) and cerebral activation during painful heat stimulation (functional scans 1 and 2). Given that temperatures were adjusted individually based on ratings, we first confirmed that differences between high and low expressive individuals were not explained by differences in stimulus intensity (mean \pm SD temperature used in low pain expression group: $48.0 \pm 0.9^{\circ} \mathrm{C}$; high pain expression group: $\left.47.8^{\circ} \mathrm{C} \pm 0.7^{\circ} \mathrm{C} ; T_{(32)}=0.79 ; p=0.43\right)$. To fur- 
ther insure that correlations between facial expressiveness and cerebral activation were not explained by differences in subjective pain ratings we also conducted a correlation analysis between facial expressiveness and self-report ratings. As expected, individual differences in facial expressiveness were not correlated with self-report ratings $(r=-0.04, p=0.82)$. This allowed us to examine brain responses associated specifically with pain expressiveness.

Facial expressiveness was positively correlated with increased BOLD signal changes in motor related structures, including face area of M1 (bilateral) and SMA (Table 3, Fig. 2). Moreover, positive correlation were also found with painrelated areas [S1 (bilateral) and bilateral posterior insula]. In contrast, lower facial expressiveness during painful trials was correlated with increased activity in the medial prefrontal cortex (MPFC), the middle frontal gyrus, and to some extent in the caudate nucleus (Table 3, Fig. 2). Follow-up analyses of the MPFC peak indicated comparable negative BOLD responses to the Pain stimulus $(t=-4,16$, $p<0.001)$ and the Warm stimulus $(t=-5.02, p<0.001$; Pain $>$ Warm: $t=-0.79, p=0.44$, nonsignificant). This is consistent with a stimulus-induced negative BOLD response in an area generally associated with the default mode network (Raichle et al., 2001). The negative correlation of this response with facial expressiveness indicates that stoic individuals displayed less "deactivation," or more sustained default mode activity, during the pain stimulus.

Pearson- $r$ correlations were also computed between the b-values obtained in the Pain $>$ Warm contrast in MPFC, the head of the caudate, and MI, as defined by the peak observed in the FACS regression, to further examine their shared variance and possibly determine mediation effects. The correlation was significant between the MPFC and the caudate $(r=0.65, p<$ $0.001)$ and between M1 and the caudate $(r=-0.49, p=0.003)$. In contrast, M1 did not correlate significantly with MPFC $(r=$ $-0.22, p=0.20$ ). Furthermore, a multiple regression model predicting FACS ranks from b-values at all three sites (stepwise) indicated at least partly independent contribution of M1 (total $\left.R^{2}=0.43, p<0.001\right)$ and MPFC $\left(\Delta R^{2}=0.18 ; \Delta p=0.001\right.$; Total $R^{2}=0.61, p<0.001$ ), while the inclusion of the caudate did not improve further the fit of the model.

\section{Within-subjects analyses of trial-by-trial fluctuations in pain} expressers (analysis 2)

For all subjects who were categorized as facially expressive $(N=21)$ as well as for slightly expressive subjects from the low/nonexpressive group $(N=7)$ (see Fig. 1), painful events were separated into trials with and without facial expressions of pain (this is possible since facial expressiveness fluctuates even within an individual across painful trials). To ensure that differences in brain activation are not due to differences in subjective pain ratings, VAS ratings were compared between events with and without facial expressions of pain; no differences were found $\left(T_{(21)}=-0.21, p=0.83\right)$.
Direct contrast between brain activation during events with and without facial expressions of pain revealed increased BOLD signal in motor-related structures, such as the face area of M1, SMA, and putamen (Table 4). Importantly, we also found stronger activation associated with the occurrence of facial expressions in pain-related areas [e.g., mostly contra-lateral S1, bilateral ACC, bilateral INS (Table 4, Fig. 3A)]. The opposite contrast revealed a very similar picture as was found for the negative correlation between facial expressiveness and cerebral activation, namely increased activity in frontal areas and the caudate nucleus (Table 4, Fig. 3A).

Effects of facial encoding of pain in nonexpressers (analysis 3) To test the hypothesis that low/nonexpressers $(N=13)$ are actively suppressing their expression at baseline (runs 1 and 2), we examined pain-related responses when they were asked to use their face to communicate how they felt during the stimulation (runs with intentionally communicated expressions, see Fig. 1). To exclude that differences in brain responses are due to differences in pain ratings, we compared subjective pain ratings between scans without/low spontaneous facial pain displays and with intentionally communicated expressions and found no differences $\left(T_{(12)}=-1.21, p=0.25\right)$.

Contrasting brain activation in scans with intentionally communicated facial expressions with scans without/with weak spontaneous facial displays revealed higher activation only in M1 (Table 5, Fig. 3B). However, the opposite contrast (no/low spontaneous pain expression $>$ intentionally communicated expressions) revealed increased BOLD response in the medial prefrontal cortex, middle frontal gyrus, as well as in the caudate nucleus (Table 5, Fig. 3B).

Thus, across all three types of analyses conducted, a very similar pattern of activation could be found when there were no or only very weak spontaneous facial expressions in response to painful stimulation. Namely, higher activation in frontostriatal 
Table 4. Within-subject analysis - differences between painful events with and without facial expressions of pain in the facially expressive individuals $(N=28)$

\begin{tabular}{|c|c|c|c|c|}
\hline \multirow[b]{2}{*}{ Brain area } & \multicolumn{3}{|c|}{ Coordinates } & \multirow{2}{*}{$\begin{array}{l}\text { Local peak } \\
t \text { value }\end{array}$} \\
\hline & $x$ & $y$ & $z$ & \\
\hline \multicolumn{5}{|c|}{ Targets of the spino-thalamocortical pathways ${ }^{a}$} \\
\hline \multirow[t]{4}{*}{ S1 } & 28 & -28 & 61 & 5.54 \\
\hline & 14 & -41 & 65 & 5.05 \\
\hline & 3 & -41 & 58 & 3.86 \\
\hline & -21 & -31 & 61 & 4.96 \\
\hline \multirow[t]{3}{*}{ S2/parietal operculum } & 65 & -24 & 17 & $3.00^{b}$ \\
\hline & -58 & -21 & 14 & 3.81 \\
\hline & -62 & -28 & 27 & 3.52 \\
\hline \multirow[t]{4}{*}{$\mathrm{ACC}$} & -3 & 21 & 27 & 2.90 \\
\hline & -10 & 0 & 41 & 3.58 \\
\hline & 7 & 10 & 41 & 3.52 \\
\hline & 0 & 7 & 41 & 3.49 \\
\hline Caudal ACC & 7 & -7 & 48 & 5.24 \\
\hline \multirow[t]{3}{*}{$\mathrm{PCC}$} & 3 & -21 & 44 & 3.66 \\
\hline & 14 & -21 & 41 & 6.32 \\
\hline & -10 & -24 & 34 & 3.58 \\
\hline \multirow[t]{3}{*}{ alNS } & 38 & 10 & 0 & $3.28^{b}$ \\
\hline & -38 & 10 & -7 & $3.37^{b}$ \\
\hline & 41 & -3 & -14 & 4.40 \\
\hline \multirow[t]{3}{*}{ pINS/claustrum } & 41 & -34 & 17 & 4.00 \\
\hline & 31 & -24 & 14 & 3.76 \\
\hline & -31 & -24 & 14 & 4.53 \\
\hline \multirow[t]{2}{*}{ Thalamus } & 10 & -17 & 10 & $3.38^{b}$ \\
\hline & -7 & -21 & -3 & $3.47^{b}$ \\
\hline \multicolumn{5}{|l|}{ Motor-related structures } \\
\hline \multirow[t]{3}{*}{ M1 } & 48 & -10 & 48 & 6.90 \\
\hline & -38 & -17 & 37 & 5.55 \\
\hline & -52 & -14 & 37 & 5.00 \\
\hline \multirow[t]{2}{*}{ SMA } & 13 & 0 & 58 & 4.33 \\
\hline & -10 & -3 & 61 & 5.01 \\
\hline \multicolumn{5}{|l|}{$\mathrm{ACC}$} \\
\hline \multicolumn{5}{|l|}{ Caudal ACC ${ }^{C}$} \\
\hline \multicolumn{5}{|l|}{ Thalamus $^{c}$} \\
\hline \multirow[t]{2}{*}{ Putamen } & 31 & -14 & 7 & $3.48^{d}$ \\
\hline & -30 & -17 & 10 & 4.59 \\
\hline \multicolumn{5}{|l|}{ Other areas } \\
\hline \multirow[t]{2}{*}{ Superior parietal gyrus } & 10 & -55 & 65 & 3.59 \\
\hline & -14 & -52 & 65 & 4.95 \\
\hline \multirow[t]{2}{*}{ Superior frontal gyrus } & 10 & 17 & 51 & 3.58 \\
\hline & -10 & 28 & 54 & 3.70 \\
\hline Globus pallidus & 34 & -7 & -3 & 4.63 \\
\hline & 27 & -7 & -3 & 3.64 \\
\hline Hippocampal gyrus & 31 & -28 & -17 & 4.34 \\
\hline & -31 & -28 & -17 & 3.55 \\
\hline Cerebellum & -14 & -58 & -20 & 4.45 \\
\hline Targets of the "expression s & & & & \\
\hline Inferior frontal gyrus & 48 & 24 & 14 & 3.52 \\
\hline Motor-related structures & & & & \\
\hline Caudate nucleus & 14 & 21 & 14 & 3.94 \\
\hline
\end{tabular}

Global search threshold: $T=3.50 . p=0.001$ (uncorrected).

${ }^{a}$ Spontaneous expression: pain expression $>$ no pain expression.

${ }^{b}$ Directed search threshold at $T=2.84(p=0.05)$. Bonferroni-corrected for 12 pain-related regions.

'See the pain expression versus no pain expression peaks.

${ }^{d}$ Marginal significance at global threshold.

eSpontaneous nonexpression: no pain expression $>$ pain expression.

circuits was observed in (1) the negative correlation with facial expressiveness across subjects, (2) trials without $>$ with spontaneous facial expressions of pain in expressive subjects, as well as (3) scans with no or only weak spontaneous expressions $>$ scans with intentionally communicated expressions in low/nonexpressive subjects. Moreover, the display of spontaneous facial expressions (analyses 1 and 2) was always coupled with increased activity in the face area of the primary motor cortex and in areas involved in pain processing.
Table 5. Within-subject analysis - differences between functional runs without spontaneous facial expressions and runs with intentionally communicated facial expressions of pain in the non/low-expressive individuals $(N=13)$

\begin{tabular}{|c|c|c|c|c|}
\hline \multirow[b]{2}{*}{ Brain area } & \multicolumn{3}{|c|}{ Coordinates } & \multirow{2}{*}{$\begin{array}{l}\text { Local peak } \\
t \text { value }\end{array}$} \\
\hline & $x$ & $y$ & $z$ & \\
\hline \multicolumn{5}{|l|}{ Targets of the "expression } \\
\hline \multicolumn{5}{|l|}{ suppression" areas $^{a}$} \\
\hline \multirow[t]{2}{*}{ Middle frontal gyrus } & 31 & 55 & 0 & 4.64 \\
\hline & -21 & 52 & 7 & 4.73 \\
\hline \multirow[t]{3}{*}{ MPFC/perigenual ACC } & 0 & 45 & -3 & 6.05 \\
\hline & 3 & 24 & -7 & 5.86 \\
\hline & -3 & 34 & -7 & 5.95 \\
\hline \multicolumn{5}{|l|}{ Motor-related structures } \\
\hline Caudate nucleus & 7 & 10 & -3 & 4.54 \\
\hline \multicolumn{5}{|l|}{ Other areas } \\
\hline Superior temporal gyrus & 62 & -14 & -3 & 4.97 \\
\hline Middle temporal gyrus & 65 & -14 & -14 & 4.17 \\
\hline \multirow[t]{2}{*}{ Precuneus } & 41 & -72 & 34 & 3.96 \\
\hline & -41 & -72 & 37 & 5.05 \\
\hline \multicolumn{5}{|l|}{ Motor-related structures $^{b}$} \\
\hline \multirow[t]{2}{*}{ M1 } & 58 & 0 & 37 & 5.47 \\
\hline & -45 & -17 & 41 & 5.44 \\
\hline
\end{tabular}

Global search threshold: $T=3.93, p=0.001$ (uncorrected)

${ }^{a}$ Spontaneous nonexpression $>$ intentionally communicated expression.

${ }^{b}$ Intentionally communicated expression $>$ spontaneous nonexpression.

\section{Discussion}

The main aim of this study was to investigate the cerebral regulation of facial expressions with a special focus on mechanisms regulating the degree of spontaneous expressiveness. We could demonstrate that low facial expressiveness (stoicism) in response to painful heat stimulation was related to higher activation in the prefrontal cortex as well as in the caudate nucleus. In contrast, the display of facial expressions of pain (or higher degrees of expressiveness) in response to painful stimulation was related to increased activity in motor-related areas [primary motor cortex (M1, face area), SMA, putamen] as well as in areas of the thalamocortical pathways that are associated with pain processing (e.g., S1, INS, ACC).

\section{Cerebral activation associated with low or no facial pain displays}

One of our strongest findings was the enhanced activity in the prefrontal areas (MPFC) and in the caudate nucleus when individuals showed spontaneously low facial responses to the painful heat stimuli. This activation pattern was found (1) when we correlated the degree of expressiveness with cortical activation (negative correlation) across all subjects; (2) when contrasting painful trials without and with spontaneous facial pain displays in facially expressive individuals, and (3) when low/nonexpressive subjects were asked to display their sensations via their facial expression and then contrasting runs with "spontaneous no/low-expression" with "intentionally communicated expression." This consistency in activation patterns found across analyses suggests strong reliability of our findings.

How can this activation pattern be interpreted? Anatomical studies in nonhuman primates as well as human studies using diffusion tensor imaging have demonstrated that the caudate nucleus, together with the putamen, have strong connections with frontal structures (e.g., medial, dorsal, ventral prefrontal cortex, frontal pole) (Alexander et al., 1990, Lehéricy et al., 2004; Leh et al., 2007). Similar findings were reported when conducting functional connectivity analysis [fMRI during rest (Di Martino et al., 2008)]. Again, the caudate nucleus was associated with activation 


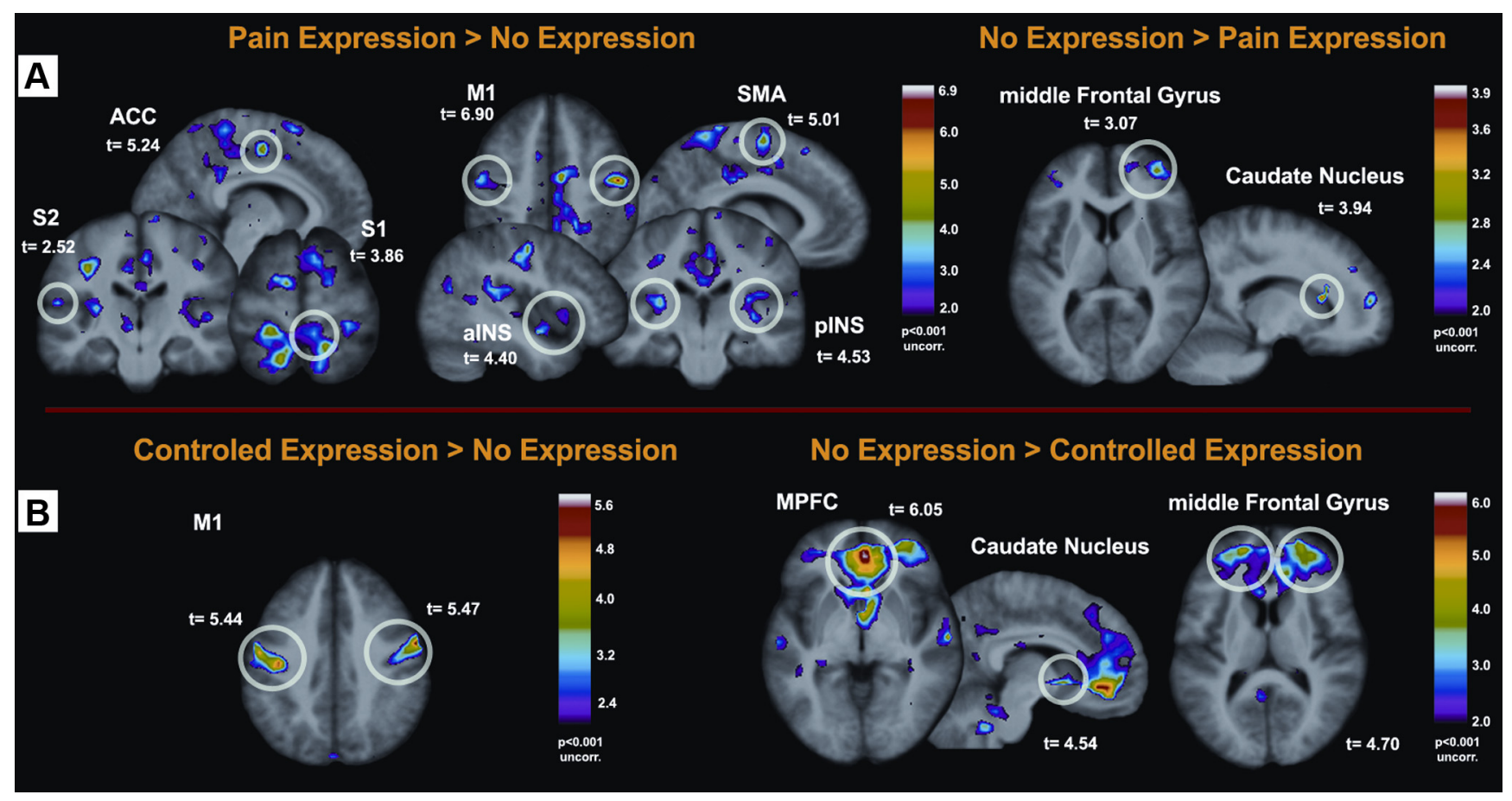

Figure 3. Within-subject analyses — differences between painful events with and without facial expressions of pain. $\boldsymbol{A}$, Stronger brain activation was found in M1 (face area) and in several pain-related areas in trials where individuals displayed spontaneous pain expression (left). In contrast, stronger activation was found in prefrontal areas and caudate nucleus when no expression was displayed (right). $\boldsymbol{B}$, In facially non/low-expressive individuals, the display of intentionally communicated facial expressions of pain was associated with increased activity in M1 (left) and reduced activity in prefrontal cortices and in the caudate nucleus (right). * Directed search threshold at $T=2.84(p=0.05)$. Bonferroni-corrected for 12 pain-related regions.

in prefrontal areas. Here, the activation peak in the head of the caudate was positively correlated to the peak in the MPFC, consistent with the activation of a frontostriatal pathway.

In line with this anatomical and functional connectivity between the caudate nucleus and prefrontal areas, numerous studies have shown that both areas are key structures within a network generally involved in response suppression and motor inhibition tasks (Rubia et al., 2001; Ridderinkhof et al., 2004; Aron et al., 2007; Boehler et al., 2010). Moreover, children with attention deficit disorder-who exhibit poorer performance on response inhibition tasks - were found to have caudate nucleus dysfunction and decreased caudate volume, as well as smaller volume of the prefrontal lobe and lower prefrontal activation (Rubia et al., 1999; Semrud-Clikeman et al., 2000; Durston et al., 2003; Cubillo et al., 2010). Thus, our findings of increased activation in prefrontal areas and in the caudate nucleus during painful events with low facial pain displays could reflect inhibitory mechanisms involved in expression suppression. This interpretation would also be in line with previous findings on emotional expressiveness. The few studies that have been conducted on this topic, namely by investigating cerebral regulation of smiles (Wild et al., 2006) and of facial responses to negative emotion-eliciting films (Goldin et al., 2008) both found BOLD activity in prefrontal areas to be related to the voluntary inhibition of emotional facial expressions. Notably, several prefrontal activation peaks found in the present study matched those found in the voluntary suppression of negative emotion in Goldin et al. (2008). In contrast, MPFC activation was only observed in the present study and may reflect a more automatic suppression process. However, without a direct within-study comparison of a variety of motor suppression conditions, it is difficult to determine whether the activation patterns we find during low facial expressiveness is specific for the suppression of facial expressions or whether it simply reflects a more general mechanism of motor inhibition.
So far, it had been unclear why some individuals show no facial expressions although they report experiencing pain. It has been argued that low expressiveness is due to active expression suppression, whereas high expressiveness is a release of suppression; but also that high expressiveness might be an exaggeration of pain behavior (for review, see Williams, 2002). Our findings provide first evidence that an absence of spontaneous facial expressions in response to pain reflect stronger activation of a brain system known to be involved in response suppression. But what are the reasons for individuals to inhibit their facial expression? Closer examination of the response observed in the MPFC might help address this question.

The association of the MPFC activity with expression suppression may relate to the role attributed to this area in self-reflective and introspective processes and associated with the default mode network (for reviews, see Northoff et al., 2006, Buckner et al., 2008). In line with this, low facial expressiveness has been linked to introspection and related personality traits [e.g., introversion (Riggio and Riggio, 2002)], and stoic individuals may maintain stronger self-related regulatory activation in this network during pain stimuli. In contrast, the behavioral responses of more expressive subjects may be driven more directly by sensory-affective processes, with less "interference" from self-reflective processes, leading to increased spontaneous facial expression. Self-reflective processes being largely conditioned by social factors (Bandura, 1991), it seems quite plausible that the MPFC, a key structure for social cognition (Lieberman, 2007), may constitute a critical node in pain and emotion expression regulation as determined by the individual implementation of social display rules.

The tendency to express or not express affective states via facial expressions develops during childhood with peers and parents generally discouraging the strong display of emotions (Izard, 1971). In line with this, Larochette et al. (2006) demonstrated that children ( $8-12$ years) were able to successfully suppress their 
facial expression of pain when asked to do so. These children reported that they sometimes suppress their pain expression in everyday life to avoid embarrassment in front of peers or to avoid worrying their parents (Larochette et al., 2006). Thus, the tendency to suppress one's facial expression of pain seems to be an early learned behavior that might be beneficial in some social contexts. However, this can also be severely disadvantageous in other settings. Since clinicians strongly rely on facial expressions when judging patients pain (Williams, 2002), the suppression of facial pain expressions (in individuals with a tendency for stoicism) can lead to underestimation of pain in health-care settings and thus to under-treatment of pain.

\section{Cerebral areas associated with the occurrence of facial pain displays}

Across all analyses, the display of facial expressions of pain was positively associated with enhanced fMRI activity in the primary motor cortex (M1; BA 4; face area, bilaterally). Interestingly, BOLD increases in M1 did not correlate with BOLD decreases in the MPFC, and multiple regression indicated that both areas contributed at least partly independently (and inversely) to the prediction of individual expressiveness, consistent with the implication of at least two separate processes. However, no significant effect was detected in the brainstem at the statistical thresholds used here, such that descending influences on the facial motor nuclei in the lower pons, or on brainstem pain regulatory systems, could not be demonstrated.

Traditionally, it was suggested that activation in cortical areas (especially M1) is mostly associated with voluntary facial expressions (Rinn, 1984). However, more recent findings postulate that distributed multifocal subcortical regions (especially basal ganglia) as well as cortical regions (especially M1) interact with each other when producing both voluntary and spontaneous facial expressions (for reviews, see Blair, 2003; Morecraft et al., 2004). Thus, finding strong activation in M1 during the display of spontaneous facial expressions of pain (Figs. 2, 3a) as well as during the display of more intentionally communicated facial responses (Fig. $3 B$ ) clearly confirms its role in both processes. Next to the strong activation in M1, spontaneous facial pain displays were also positively associated with stronger responses in SMA and the putamen, consistent with earlier reports (Blair, 2003). Interestingly, during the occurrence of facial expressions we also observed higher activation in areas of cortical targets of the spinothalamic pathways implicated in the processing of pain. These include primary somatosensory area (leg area contralateral to the stimulation side), insula, and ACC. This increased BOLD activity in pain-related areas was most pronounced in the withinsubject analyses comparing trials with and without facial expressions in facially expressive subjects (Table 3 ). This effect could be interpreted in two complementary ways. First, self-report ratings were not correlated with facial expressiveness and did not differ between painful trials with and without facial expressions, so that the variability in facial responses and the corresponding brain responses cannot be explained by differences in pain reports. Facial expression of pain therefore appears to capture unique aspects of nociceptive processing within the cortical targets of the spino-thalamocortical pathway. Given the well accepted role of this system in pain perception, this implies that facial expression reflects some aspects of pain experiences not fully captured by self-report, consistent with previous behavioral and clinical interpretations (Craig et al., 2001). A second interpretation suggests that the inhibitory mechanisms involved in the downregulation of facial expressions might produce a more general downregulation of cerebral pain processing. Thus, activity in pain-related areas might decrease while inhibiting one's facial expression. Following this line of reasoning, the suppression of facial expressions might reflect a regulatory mechanism that decreases cerebral activation in pain-related areas but that is not captured in self-report measures. However, the present results did not provide direct evidence that MPFC activation, reflecting expression suppression, was related to the activation of pain-related inhibitory processes. These interpretations are not mutually exclusive and both support the valid contribution of facial expression measures to assess pain processing.

\section{Conclusions}

Facial expressions of emotions play a crucial role in interindividual interactions. Interestingly, however, individuals differ substantially in their tendency to encode their affective state via this major communication channel. Results indicated that facial pain displays do reflect at least in part the activity within nociceptive pathways that is not fully captured by pain reports; while stoicism — as indicated by frontostriatal activation-involves the active suppression of expression. Differences in facial expressiveness have been discussed in the context of learned display rules regulating when and how one should express specific affective states. Our data clearly demonstrates the involvement of frontostriatal circuits in the downregulation of facial expressiveness. We propose that activity within this brain network reflects the implementation of expression display rules, and that this system is integral to the neurobiological machinery underlying the regulation of complex social interactions.

\section{References}

Alexander GE, Crutcher MD, DeLong MR (1990) Basal gangliathalamocortical circuits: parallel substrates for motor, oculomotor, "prefrontal" and "limbic" functions. Prog Brain Res 85:119-146.

Apkarian AV, Bushnell MC, Treede RD, Zubieta JK (2005) Human brain mechanisms of pain perception and regulation in health and disease. Eur J Pain 9:463-484.

Aron AR, Durston S, Eagle DM, Logan GD, Stinear CM, Stuphorn V (2007) Converging evidence for a fronto-basal-ganglia network for inhibitory control of action and cognition. J Neurosci 27:11860-11864.

Bandura A (1991) Social cognitive theory of self-regulation. Organ Behav Hum Decis Process 50:248-287.

Blair RJ (2003) Facial expressions, their communicatory functions and neuro-cognitive substrates. Philos Trans $\mathrm{R}$ Soc Lond B Biol Sci 358:561-572.

Boehler CN, Appelbaum LG, Krebs RM, Hopf JM, Woldorff MG (2010) Pinning down response inhibition in the brain - conjunction analyses of the Stop-signal task. Neuroimage 52:1621-1632.

Buckner RL, Andrews-Hanna JR, Schacter DL (2008) The brain's default network: anatomy, function, and relevance to disease. Ann N Y Acad Sci 1124:1-38.

Craig KD, Prkachin KM, Grunau RVE (2001) The facial expression of pain. In: Handbook of pain assessment, Ed 2 (Turk DC, Melzack R, Eds), pp 153-169. New York: Guilford.

Cubillo A, Halari R, Ecker C, Giampietro V, Taylor E, Rubia K (2010) Reduced activation and inter-regional functional connectivity of fronto-striatal networks in adults with childhood Attention-Deficit Hyperactivity Disorder (ADHD) and persisting symptoms during tasks of motor inhibition and cognitive switching. J Psychiatr Res 44:629-639.

Darwin C (1872) The expression of the emotions in man and animals. Chicago: University of Chicago.

Di Martino A, Scheres A, Margulies DS, Kelly AM, Uddin LQ, Shehzad Z, Biswal B, Walters JR, Castellanos FX, Milham MP (2008) Functional connectivity of human striatum: a resting state FMRI study. Cereb Cortex 18:2735-2747.

Dum RP, Levinthal DJ, Strick PL (2009) The spinothalamic system targets motor and sensory areas in the cerebral cortex of monkeys. J Neurosci 29:14223-14235. 
Durston S, Tottenham NT, Thomas KM, Davidson MC, Eigsti IM, Yang Y, Ulug AM, Casey BJ (2003) Differential patterns of striatal activation in young children with and without ADHD. Biol Psychiatry 53:871-878.

Ekman P (1999) Facial expressions In: The handbook of cognition and emotion (Dalgleish, T, Power, T, eds), pp 45-60. Sussex, UK: Wiley.

Ekman P, Friesen W (1969) The repertoire of nonverbal behavior: categories, origins, usage, and coding. Semiotica 1:49-98.

Ekman P, Friesen WV (1987) Facial action coding system. Palo Alto, CA: Consulting Psychologists.

Elfenbein HA, Beaupré MG, Lévesque M, Hess U (2007) Toward a dialectic theory: cultural differences in the expression and recognition of posed facial expressions. Emotion 7:131-146.

Friston KJ, Holmes AP, Worsley KJ, Poline JB, Frith CD Frackowiak RSJ (1995) Statistical parametric maps in functional imaging: a general linear approach. Hum Brain Mapp 2:189-210.

Fusar-Poli P, Placentino A, Carletti F, Landi P, Allen P, Surguladze S, Benedetti F, Abbamonte M, Gasparotti R, Barale F, Perez J, McGuire P, Politi P (2009) Functional atlas of emotional faces processing: a voxel-based meta-analysis of 105 functional magnetic resonance imaging studies. J Psychiatry Neurosci 34:418-432.

Goldin PR, McRae K, Ramel W, Gross JJ (2008) The neural bases of emotion regulation: reappraisal and suppression of negative emotion. Biol Psychiatry 63:577-586.

Gross JJ, Levenson RW (1993) Emotional suppression: physiology, selfreport, and expressive behaviour. J Pers Soc Psychol 64:970-986.

Halberstadt AG, Denham SA, Dunsmore JC (2001) Affective social competence. Soc Development 10:79-119.

Iwase M, Ouchi Y, Okada H, Yokoyama C, Nobezawa S, Yoshikawa E, Tsukada H, Takeda M, Yamashita K, Takeda M, Yamaguti K, Kuratsune H, Shimizu A, Watanabe Y (2002) Neural substrates of human facial expression of pleasant emotion induced by comic films: a PET study. Neuroimage 17:758-768.

Izard CE (1971) The face of emotion. New York: Appleton-Century-Crofts.

Kunz M, Gruber A, Lautenbacher S (2006) Sex differences in facial encoding of pain. J Pain 7:915-928.

Kunz M, Scharmann S, Hemmeter U, Schepelmann K, Lautenbacher S (2007) The facial expression of pain in patients with dementia. Pain 133:221-228

Kunz M, Chatelle C, Lautenbacher S, Rainville P (2008) The relation between catastrophizing and facial responsiveness to pain. Pain 140:127-134.

Larochette AC, Chambers CT, Craig KD (2006) Genuine, suppressed and faked facial expressions of pain in children. Pain 126:64-71.

Leh SE, Ptito A, Chakravarty MM, Strafella AP (2007) Fronto-striatal connections in the human brain: a probabilistic diffusion tractography study. Neurosci Lett 419:113-118.

Lehéricy S, Ducros M, Van de Moortele PF, Francois C, Thivard L, Poupon C, Swindale N, Ugurbil K, Kim DS (2004) Diffusion tensor fiber tracking shows distinct corticostriatal circuits in humans. Ann Neurol 55:522-529.

Lieberman MD (2007) Social cognitive neuroscience: A review of core processes. Annu Rev Psychol 58:259-289.
Morecraft RJ, Stilwell-Morecraft KS, Rossing WR (2004) The motor cortex and facial expression: new insights from neuroscience. Neurologist 10:235-249.

Northoff G, Heinzel A, de Greck M, Bermpohl F, Dobrowolny H, Panksepp J (2006) Self-referential processing in our brain - a meta-analysis of imaging studies on the self. Neuroimage 31:440-457.

Ogawa S, Lee TM, Kay AR Tank DW (1990) Brain magnetic resonance imaging with contrast dependent on blood oxygenation. Proc Natl Acad Sci U S A 87:9868-9872.

Raichle ME, MacLeod AM, Snyder AZ, Powers WJ, Gusnard DA, Shulman GL (2001) A default mode of brain function. Proc Natl Acad Sci U S A 98:676-682.

Richards JM, Gross JJ (2000) Emotion Regulation and Memory: the cognitive costs of keeping one's cool. J Pers Soc Psychol 79:410-424.

Ridderinkhof KR, van den Wildenberg WP, Segalowitz SJ, Carter CS (2004) Neurocognitive mechanisms of cognitive control: the role of prefrontal cortex in action selection, response inhibition, performance monitoring, and reward-based learning. Brain Cogn 56:129-140.

Riggio HR, Riggio RE (2002) Extraversion, neuroticism, and emotional expressiveness: a meta-analysis. J Nonvl Behav 26:195-218.

Rinn WE (1984) The neuropsychology of facial expression-a review of neurological and psychological mechanisms for producing facial expressions. Psychol Bull 95:52-77.

Rubia K, Overmeyer S, Taylor E, Brammer M, Williams SC, Simmons A, Bullmore ET (1999) Hypofrontality in attention deficit hyperactivity disorder during higher-order motor control: a study with functional MRI. Am J Psychiatry 156:891-896.

Rubia K, Russell T, Overmeyer S, Brammer MJ, Bullmore ET, Sharma T, Simmons A, Williams SC, Giampietro V, Andrew CM, Taylor E (2001) Mapping motor inhibition: conjunctive brain activations across different versions of go/no-go and stop tasks. Neuroimage 13:250-261.

Semrud-Clikeman M, Steingard RJ, Filipek P, Biederman J, Bekken K, Renshaw PF (2000) Using MRI to examine brain-behavior relationships in males with attention deficit disorder with hyperactivity. J Am Acad Child Adolesc Psychiatry 39:477-484.

Tsao DY, Livingstone MS (2008) Mechanisms of face perception. Annu Rev Neurosci 31:411-437.

Weddell RA (1994) Effects of subcortical lesion site on human emotional behavior. Brain Cogn 25:161-193.

Weddell RA, Miller JD, Trevarthen C (1990) Voluntary emotional facial expressions in patients with focal cerebral lesions. Neuropsychologia 28:49-60

Weddell RA, Trevarthen C, Miller JD (1988) Reactions of patients with focal cerebral lesions to success or failure. Neuropsychologia 26:373-385.

Wild B, Rodden FA, Rapp A, Erb M, Grodd W, Ruch W (2006) Humor and Smiling: Cortical Areas Selective for Cognitive, Affective and Volitional Components. Neurology 66:887-893.

Williams AC (2002) Facial expression of pain: an evolutionary account. Behav Brain Sci 25:439-455. 